\title{
Bangla/English Script Identification Based on Analysis of Connected Component Profiles
}

\author{
Lijun Zhou ${ }^{1}$, Yue $\mathrm{Lu}^{1,2}$, and Chew Lim $\operatorname{Tan}^{3}$ \\ 1 Department of Computer Science and Technology, \\ East China Normal University, Shanghai 200062, China \\ 2 Shanghai Research Institute of Postal Science, \\ China State Post Bureau, Shanghai 200062, China \\ 3 Department of Computer Science, School of Computing, \\ National University of Singapore, Kent Ridge, Singapore 117543
}

\begin{abstract}
Script identification is required for a multilingual OCR system. In this paper, we present a novel and efficient technique for Bangla/English script identification with applications to the destination address block of Bangladesh envelope images. The proposed approach is based upon the analysis of connected component profiles extracted from the destination address block images, however, it does not place any emphasis on the information provided by individual characters themselves and does not require any character/line segmentation. Experimental results demonstrate that the proposed technique is capable of identifying Bangla/English scripts on the real Bangladesh postal images.
\end{abstract}

\section{Introduction}

Language identification acts as an important role in document image processing, especially for multi-lingual OCR systems. Its goal is to automatically classify textual document images, based on analyzing the stroke structure and connections and the fundamentally different writing styles of the different alphabets or character sets. In past years, many algorithms for script identification have been proposed. According to entities analyzed in the process of script identification, the algorithms proposed in the literature could be typically classified to four categories: (a) the schemes based on analysis of connected components [1-2]. (b) the schemes based on analysis of characters, words and text lines[3-7]. (c) the schemes based on analysis of text blocks[8-10]. (d) the schemes based on analysis of hybrid information of connected components, text lines etc.[11-16]. We discuss briefly the principles, merits and weakness of each approach.

\subsection{Connected Component Analysis}

The approaches based on connected component analysis generally use the intrinsic morphological characteristics of the character sets or strokes of each script. Hochberg et al. [1] presented a system that automatically identifies the 
script form using cluster-based templates. It discovers frequent character or word shapes in each script by means of cluster analysis, then looks for instances of these in new documents and compares a subset of textual symbols from the document to each script's templates. The script with the best match is chosen as the script of the document.

In [2], Spitz presented an approach for automatic determination of the script and language content of document images on the basis of character density or the optical distribution. Based on the spatial relationships of features related to the upward concavities in character structures, the method first classifies the script into two broad classes: Han-based and Latin-based. Language identification within the Han script class (Chinese, Japanese, Korean) is performed by analysis of the distribution of optical density in the text images. They handled 23 Latin-based languages using a technique based on character shape codes.

\subsection{Character, Word or Line Analysis}

Most methods based on the analysis of character, word or line have been proposed for language identification in multilingual documents. Lee and Kim [3] proposed a scheme for multi-lingual, multi-font, and multi-size large-set character recognition using self-organizing neural network. They determine not the script of the entire document, but the script of individual characters within the document. In [4], Ying et al. carried out language identification by classifying individual character images to determine the language boundaries in multilingual documents.

In [5], John presented Linguini, a vector-space based categorizer used for language identification. Linguini uses dictionaries generated from features extracted from training texts, and compares these against feature vectors generated from test inputs. Features used are N-grams and words, and combinations of both. They also presented an algorithm for detecting and determining the nature of bilingual documents.

In [6], three efficient techniques for identifying Arabic script and English script were presented and evaluated. These techniques address the language identification problem on the word level and on textline level. The characteristics of horizontal projection profiles as well as run-length histograms for text written in both languages are the basic features underlying these techniques.

Tan et al. [7] presented a research in identifying English, Chinese, Malay and Tamil in image documents. The identification process takes place in two main steps. The first step uses bounding boxes of character cells and upward concavities to distinguish between three main classes: Chinese, Latin and Tamil scripts. Then if Latin scripts are detected, they use statistical analysis of word shape tokens of the Latin words in the document to distinguish between English and Malay languages.

\subsection{Text Block Analysis}

Since visual appearances of different scripts are often distinctive from each other, a text block in each script class may be considered as a unique texture 
pattern. Thus, texture classification algorithms may be employed to perform script identification. Such texture based approach is presented in [8, 9]. In [8], Peake et al. presented a new scheme based on texture analysis for script identification which did not require character segmentation. Via simple processing, a uniform text block on which texture analysis can be performed is obtained from a document image. Multiple channel (Gabor) filters and grey level co-occurrence matrices are used in order to extract texture features. They used the K-NN classifier to classify the test documents. In [9], Singhal et al. proposed an approach on script-based classification of handwritten text documents in a multilingual environment. They apply denoising, thinning, pruning, m-connectivity and text size normalization in sequence to produce a unique text block. They also use Multi-channel Gabor flltering to extract text features.

Wood et al. [10] proposed a scheme for determining the language classification of printed documents. In that algorithm, the characteristics on the horizontal and vertical projections of the document are used to distinguish European languages, Russian, Arabic, Chinese, and Korean.

\subsection{Hybrid Analysis}

Most comparatively complex methods are based on hybrid feature analysis. These schemes try to combine the different features extracted from global (text block) and local (text line, word, character and connected components) document entities.

In [12], Pal and Chaudhuri used projection profiles, statistical, topological and stroke based features for identifying English, Urdu, Bangla and Devanagari scripts from a document image. Their work was extended to separation of printed Roman, Chinese, Arabic, Devnagari, and Bangla text lines from a single document[13]. Shape based features, statistical features and some features obtained from the concept of water reservoir, have been used in this technique.

In [15], Chaudhury et al. proposed three trainable classification schemes for identification of Indian scripts. The first scheme is based upon a frequency domain representation of the horizontal profile of the textual blocks. The other two schemes use connected components extracted from the textual region. They have proposed a novel Gabor filter-based feature extraction scheme for the connected components. They also use frequency distribution of the width-to-height ratio of the connected components for script recognition. It is claimed that the Gabor filter-based scheme provides the most reliable performance.

The methods discussed above are summarized in table 1, from which we can notice that very few works have been done in identification of handwritten document images compared to machine generated document images. Most of the script identification techniques available in the literature so far consider printed text only. These techniques, especially those schemes that are based on the overall visual appearance of the text block, are generally incapable of tackling the variations in the writing style, character style and size, spacing between lines/words, etc. 
China Post is designing and manufacturing automatic letter sorting machine for Bangladesh Post Office. As a multi-language country, Bangladesh envelopes may be handwritten/printed in Bangla or English. To automatically recognize postcodes or address, Bangla/English script identification in the destination address block (DAB) becomes a crucial step. However, we found that the reported approaches are generally not suitable for our purpose. This is because all these methods apply to a wider range of languages while we specifically would like to maximize the discriminating capability between the Bangla and English scripts for this practical application. In this paper, we propose a novel connected component analysis based approach to identifying Bangla/English scripts on both printed and handwritten envelope images.

Table 1. Summarization of the methods on script identification

\begin{tabular}{|c|c|c|}
\hline Method & Language/Script & Nature \\
\hline $\begin{array}{l}\text { Cluster-Based } \\
{[1]}\end{array}$ & $\begin{array}{l}\text { Arabic, Armenian, Burmese, Chinese. } \\
\text { Cyrillic, Devanagari, Ethiopic, Greek, } \\
\text { Hebrew, Japanese,Korean, Roman, Thai }\end{array}$ & Printed \\
\hline $\begin{array}{l}\text { Analysis of character } \\
\text { density or the optical } \\
\text { distribution [2] }\end{array}$ & $\begin{array}{l}\text { Han script class(Chinese, Japanese, } \\
\text { Korean), } 23 \text { Latin-based languages }\end{array}$ & Printed \\
\hline $\begin{array}{ll}\text { Self-organizing } & \text { neural } \\
\text { network [3] } & \\
\end{array}$ & English, Korean and Chinese & Printed \\
\hline $\begin{array}{l}\text { The } r \text { prototype } \\
\text { classification method and } \\
\text { support vector machines } \\
{[4]}\end{array}$ & Chinese, English and Japanese & Printed \\
\hline Linguini $[5]$ & $\begin{array}{llr}\text { Catalan, Danish, Dutch, English, } & \text { Finnish } \\
\text { French, German, Icelandic, } & \text { Italian } \\
\text { Norwegian, Portuguese, Spanish Swedish }\end{array}$ & Printed \\
\hline $\begin{array}{l}\text { Horizontal projection } \\
\text { profiles and run-length } \\
\text { histograms analysis [6] }\end{array}$ & Arabic, English & Printed \\
\hline $\begin{array}{l}\text { Analysis of bounding } \\
\text { boxes of character cells } \\
\text { and statistical analysis of } \\
\text { word shape [7] }\end{array}$ & English, Chinese, Malay and Tamil & Printed \\
\hline Texture analysis $[8]$ & $\begin{array}{llll}\text { Chinese, } & \text { English, } & \text { Greek, } & \text { Korean, } \\
\text { Malayalam, } & \text { Persian and Russian } & \end{array}$ & Printed \\
\hline \begin{tabular}{|ll} 
Texture & classification \\
algorithm [9] & \\
\end{tabular} & Roman, Devanagari, Bangla and Telugu & Handwritten \\
\hline $\begin{array}{l}\text { Horizontal and vertical } \\
\text { projections analysis[10] }\end{array}$ & $\begin{array}{l}\text { European languages, Russian, Arabic, } \\
\text { Chinese, and Korean }\end{array}$ & Printed \\
\hline Hybrid analysis [12-15] & Indian scripts, Roman, Chinese, Arabic & Printed \\
\hline $\begin{array}{l}\text { Morphological analysis } \\
\text { combined with geometrical } \\
\text { analysis[16] }\end{array}$ & Arabic and Latin scripts & $\begin{array}{l}\text { Printed and } \\
\text { handwritten }\end{array}$ \\
\hline
\end{tabular}




\section{Proposed Technique for Script Identification}

At the first step, the grey scale image is captured from the envelope at the resolution of 200DPI, while a letter is passing by the camera on a letter sorting machine. An adaptive threshold approach is utilized to convert the grey scale image to its binary one, as given in Fig.1(a) and (b). The postal stamp block and other graphic parts are detected and deleted. Such processing is a basic stage, but out of the scope of this paper, and we will not report the corresponding details here. Based on the positional information of the text block, the DAB is extracted for subsequent processing as showed in Fig.1(c) and (d).

For language identification, choosing appropriate features is an important perhaps the most important step. For our purpose, the features used for distinguishing Bangla script and English script are chosen with the following considerations: (a) Easy to detect; (b) Feasible for identification; (c) Independence of font, size and style of the text; (d) Robustness.

The basic alphabets of English and Bangla are shown in Fig.2, from where we can note that English characters are symmetric and regular in the pixel distribution in the vertical direction whereas the difference in the vertical pixel distribution of Bangla characters is prominent. For the English characters, both the location of the lowest and the topmost pixels of each column of the

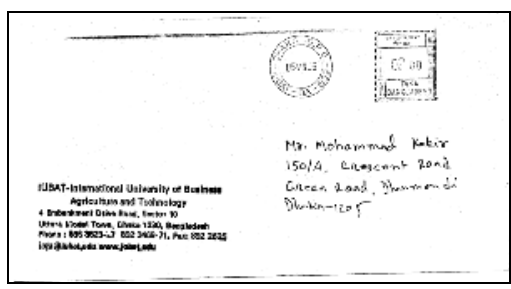

(a)

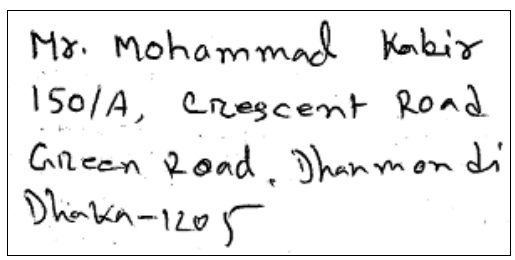

(c)

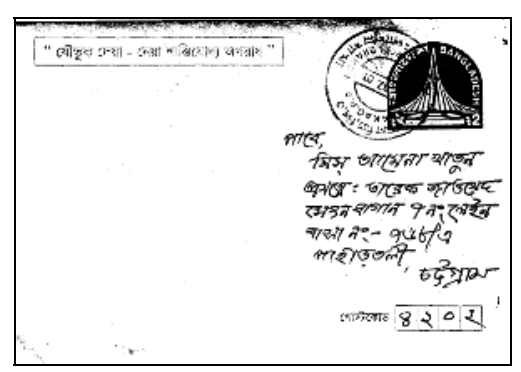

(b)

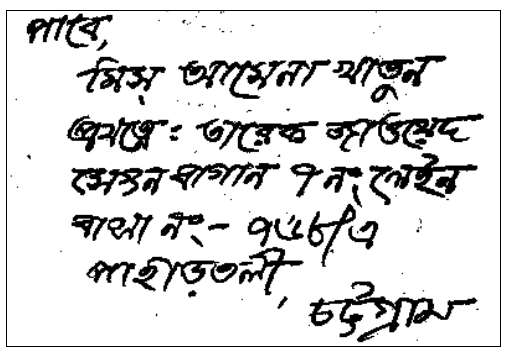

(d)

Fig. 1. (a) An example of envelop image written in English. (b) An example of envelop image written in Bangla. (c) Detected destination address block of (a). (d) Detected destination address block of (b). 


\section{abcdefghijklmnopqrstuvwxyz A B C DEF GHIJKLMNOP QR T U VW X Y Z}

(a)

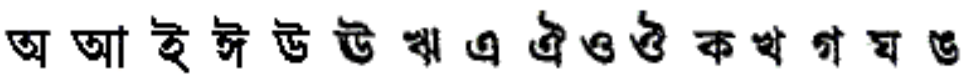

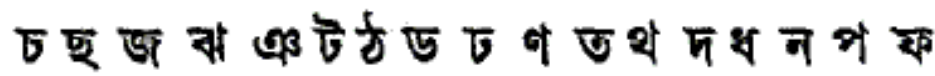

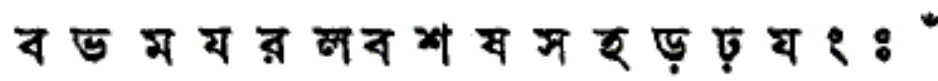

(b)

Fig. 2. (a) Basic alphabets of English (b) Basic alphabets of Bangla

components vary regularly. In Bangla, it is noted that many characters of these alphabets have a horizontal line at the upper part which is called the head-line. When two or more characters sit side by side to form a word in this language, the head-line portions touch one another and generate a long head-line. Most of the pixels of the head-line are the topmost pixels of vertical columns of the components. This kind of line, however, is absent in the lower part. It results in the distinction between the fluctuations (warps) of the topmost and the lowest pixels in each column (top and bottom profile) of the components. Thus, we can take this characteristic as a feature to distinguish English script and Bangla script. As we observed, such feature is weakened on handwritten textual document images, however, it is still sufficient for identification.

\subsection{Connected Components Labelling}

To extract features from the text block, the set of connected components in the DAB image is calculated first. Since Bangla text is cursive i.e. characters are connected within each word, a connected component in Bangla text image may correspond to a word. In contrast, English characters are isolated unless conditions due to low print quality or poor scanning. Thus, a connected component is generally related to a character in printed English text block. In the cases of handwritten text blocks, both English and Bangla, a connected component may correspond to either a character or a word, or several characters within a word owing to different writing styles of different people. However, this doesn't affect the performance of our proposed scheme.

\subsection{Meaningful Connected Components Selection}

In order to minimize the effect of non-script specific markings and reduce the computational time as well, during the analysis of the connected components 
profiles (both topmost profile and bottommost profile), absolutely very small elements, relatively very small or large elements are eliminated. This ensures that we consider only meaningful connected components and at the same time we can avoid special noise appearing in the text.

Absolutely very small elements deletion: To select meaningful connected components, we firstly deleted those with small area, currently set at less than 9 pixels. This processing removes noise and assures the veracity of the average area (amount of pixels) of textual components, which is computed as

$$
a v g=\frac{1}{M} \sum_{i=1}^{M} p i x(i)
$$

where $M$ is the number of the remaining connected components in the destination address block.

Relatively small elements deletion: Based on the considerations that most relatively small connected components correspond to punctuations or broken parts of characters or strokes which will affect the accuracy of the script identification, the components that are smaller than a predefined threshold should be excluded from further feature analysis. And the corresponding $T_{s}$ is defined as

$$
T_{s}=\alpha_{1} \times a v g
$$

In our experiment, $\alpha_{1}=0.6$ has been proved to be appropriate.

Relatively large elements deletion: As during the step of destination address block extraction, part of the postal stamp may be included because of the overlapping of them. Also, scratched-out words may be sometimes involved in the DAB. These parts, comparatively large, should be removed too. Here we considered the components which are larger than a threshold $T_{l}$ as large components. In other words, the component whose area is larger than $T_{l}$ is eliminated from the profile analysis. $T_{l}$ is computed as

$$
T_{l}=\alpha_{2} \times a v g
$$

where $\alpha_{2}=5$ has been proved to be a suitable value.

Based on the above processing, the results of connected components filtration of Fig.1(c) and (d) are shown in Fig.3(a) and (b), respectively.

\subsection{Connected Component Profiles Analysis}

Subsequently, we extract the topmost profile and the bottommost profile of the finally remained connected components respectively, i.e. the topmost pixels and the lowest pixels of vertical columns of the components. To obtain the topmost (bottommost) profile, each vertical column of a particular connected component is scanned from top (bottom) until it reaches a black pixel $\left(p_{i}\right)$. Thus, for a component of width $N$, we get $N$ such pixels. The topmost profile 


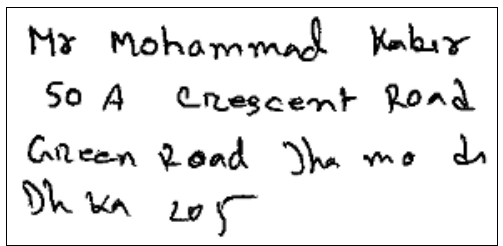

(a)

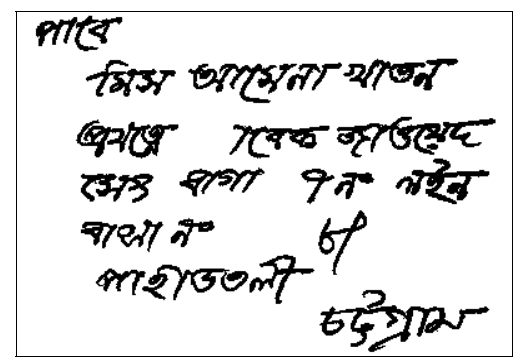

(b)

Fig. 3. (a) Connected components filtered of Fig.1(c). (b) Connected components filtered of Fig.1(d).

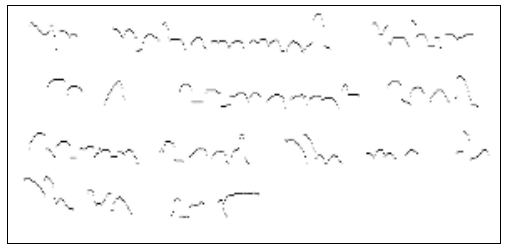

(a)

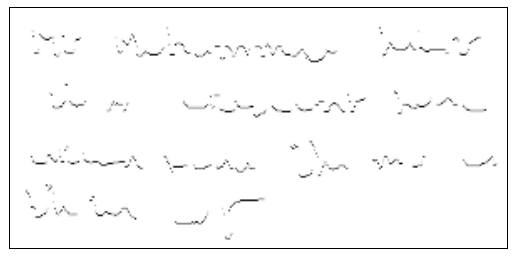

(c)

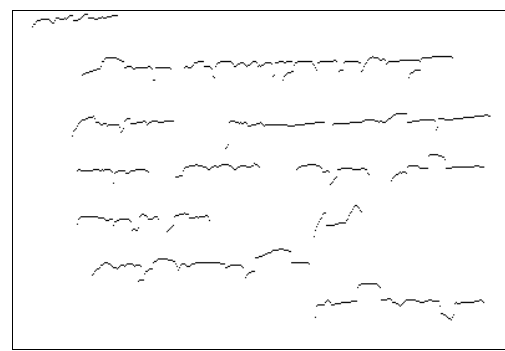

(b)

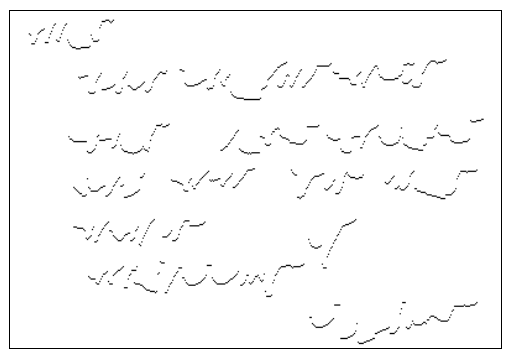

(d)

Fig. 4. (a) Topmost profile of Fig.3 (a). (b) Topmost profile of Fig.3 (b). (c) Bottommost profile of Fig.3 (a). (d) Bottommost profile of Fig.3 (b).

and bottommost profile of Fig.3.(a) and (b) are showed in Fig.4. To measure the discontinuity of topmost (bottommost) contour line of the component, we traverse from $p_{i}$ to $p_{i+1}$, and obtain the difference $d_{i}$ of two adjacent pixels of the components, and is computed as:

$$
d_{i}=\left|y_{p_{i+1}}-y_{p_{i}}\right|, \quad 1 \leq i \leq N-1
$$

where $y_{p_{i}}$ is the Y-coordinate value of the pixel $p_{i}$. 
And the total distance of the top border of the component is computed as

$$
t d(j)=\sum_{i=1}^{N-1} d_{i}
$$

On the assumption that the text block has $M^{\prime}$ connected components, its aggregate value of distance of top pixels is produced as

$$
t t d=\sum_{j=1}^{M^{\prime}} t d(j)
$$

The aggregate distance of the bottom pixels, then, is obtained in the similar way and is computed as

$$
t b d=\sum_{j=1}^{M^{\prime}} b d(j)
$$

where $b d(j)$ is the accumulative difference of bottom pixels of a connected component which is produced like the $t d(j)$ term. Text script is inferred from functions $t t d$ and $t b d$, on the basis that an English text image will have almost equal value of $t t d$ and $t b d$ whereas the difference in $t t d$ and $t b d$ is obviously large in Bangla text image. A normalized measure of this top/bottom difference $D_{t b}$ is defined as

$$
D_{t b}=\frac{t t d-t b d}{\min (t t d, t b d)}
$$

which is generally indicative of an English text block when positive, and a Bangla text block when appeared to be negative. Exception exists in text blocks when the $D_{t b}$ term of English text blocks is negative. However, we also find that the absolute value of $D_{t b}$ in both printed and handwritten English text image is generally small. In contrast, the absolute value of $D_{t b}$ in Bangla text image, either printed text or handwritten text, is comparatively large. To investigate we have done an experiment using 100 images (including English text blocks and Bangla text blocks) which are segmented from the real envelope images provided by Bangladesh Post. Through the experiment, two threshold-value are adopted which have been proved to be suitable, the one is thresh $1=0.3$, another is thresh $2=0.1$. We identify the script according to the following rules:

Rule 1: if $D_{t b}$ is larger than thresh1, the script of the text block is identified as Bangla.

Rule 2: if $D_{t b}$ is smaller than thresh2, the script of the text block is identified as English.

Rule 3: otherwise, the image is rejected from script identification.

The confidence level of the identification increases with increasing difference between $D_{t b}$ and the threshold-value. 


\section{$3 \quad$ Experimental Results}

1200 images have been used for test in our experiments. These samples were captured from real Bangladesh postal images. A small amount of page skew was inevitably introduced in practical environment and the character sizes and writing styles were vastly different. Some samples are showed in Fig.5.

The experimental results are shown in Table 2 and Table 3 . It is observed that the accuracy of script identification is very high for printed text, and for handwritten text, the proposed approach can also achieve a satisfactory accuracy of about $95 \%$.

From the experiments, we noticed that the main reason of mis-recognition and rejection are poor quality of envelope images. And the erroneous identifications of English script were found to be mostly due to the lower part connection of
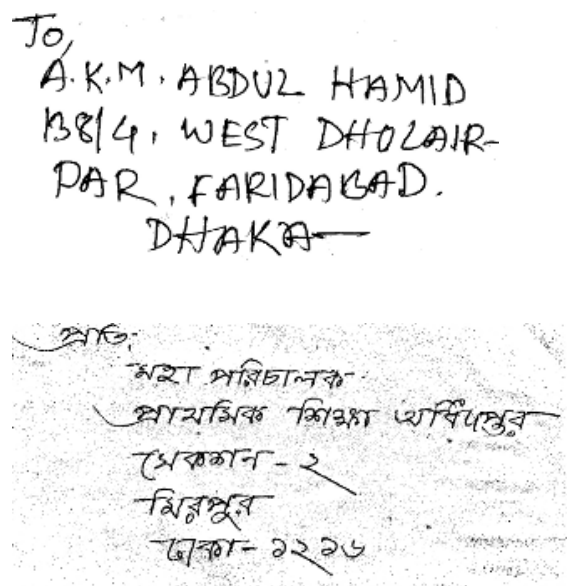

\section{里o}

DR.MD. SOHRAB ALI,

Vi11. Barodewra P.0. M18hatnagar,

P. S. Tungi

Dist.Gazipur Bangladesh.

Fig. 5. Examples of destination address block used in the experiment

Table 2. Performance for identifying printed envelope images

\begin{tabular}{|l|l|l|l|}
\hline & \multicolumn{2}{|c|}{ Recognized as } & \\
\hline Script & English & Bangla & Rejected \\
\hline English & 98.00 & 0.66 & 1.33 \\
\hline Bangla & 0 & 100.00 & 0 \\
\hline
\end{tabular}

Table 3. Performance for identifying handwritten envelope images

\begin{tabular}{|l|l|l|l|}
\hline & \multicolumn{2}{|c|}{ Recognized as } & \\
\hline Script & English & Bangla & Rejected \\
\hline English & 94.67 & 1.33 & 4.00 \\
\hline Bangla & 0 & 95.33 & 4.67 \\
\hline
\end{tabular}


the characters or words. As the lower part of the components is connected and the upper part of the components is unconnected, the distance of the topmost pixels increases whereas the distance of the bottom pixels decrease. However, this seldom occurs.

\section{Conclusions}

In this paper, we present a simple but novel technique for script identification with applications to the destination address block of Bangladesh envelope images. The approach is based upon the analysis of connected component profiles, however, it does not place any emphasis on the information provided by individual characters themselves and does not require any character/line segmentation. During the extraction of features characterizing the visual appearance of the destination address block, special connected components that are either too small or too large are deleted prior to feature analysis. Thus, the approach is robust with respect to noise. It is clear that this approach is insensitive to character size, font, writing style and case variation in the destination address block. Also, the approach is immune from text height, inter-line, inter-word spacings and skew. Experimental results have showed that relatively simple technique can reach a high accuracy level for discriminating among English script and Bangla script.

\section{Acknowledgements}

This work is jointly supported by the National Natural Science Foundation of China under grant no. 60475006, and the Contract Project of Bangladesh Post No.PARI-2/2-16/2003/2004.

\section{References}

1. J. Hochberg, P. Kelly, T. Thomas, L. Kerns, Automatic Script Identification From Document Images Using Cluster-Based Templates, IEEE Transactions on Pattern Analysis and Machine Intelligence, pp. 176-181,1997

2. A. L. Spitz, Determination of the Script and Language Content of Document Images, IEEE Trans. Pattern Analysis and Machine Intelligence, pp. 235-245, 1997

3. S. W. Lee, J. S. Kim, Multi-lingual, multi-font and multi-size large-set character recognition using self-organizing neural network, Proceedings of International Conference on Document Analysis and Recognition, Vol.1, pp. 28-33, 1995

4. Y. H. Liu, C. C. Lin, F. Chang, Language Identification of Character Images Using Machine Learning Techniques, Proceedings of 8th Intl. Conf. Document Analysis and Recognition, pp. 630-634, 2005

5. M. P. John, Linguini: Language Identification for Multilingual Documents, Proceedings of 32nd Hawaii International Conference on System Sciences, vol.2, pp. 2035-2045, 1999 
6. A. M. Elgammal, M. A. Ismail, Techniques for Language Identification for Hybrid Arabic-English Document Images, IEEE Proceedings of the Sixth International Conference on Document Analysis and Recognition, pp.1100-1104, 2001

7. C. L. Tan, T Y Leong and S He, Language identification in multilingual documents, Proceedings of International Symposium on Intelligent Multimedia and Distance Education (ISIMADE'99), pp.59-64, 1999

8. G. S. Peake, T.N. Tan, Script and Language Identification from Document Images, Proceedings of the Workshop on Document Image Analysis, pp.10-17, 1997

9. V. Singhal, N. Navin, D. Ghosh, Script-based classification of Hand-written Text Document in a Multilingual Environment, Research Issues in Data Engineering, pp.47-54, 2003

10. S. L. Wood, Xiaozhong Yao, K. Krishnamurthi, L. Dang, Language identification for printed text independent of segmentation, Proceedings of the International Conference on Image Processing, vol.3, pp.3428-3431, 1995

11. J. Ding, L. Lam, Ching Y. Suen, Classification of Oriental and European Scripts by Using Characteristic Features, Proceedings of fourth International Conference Document Analysis and Recognition, pp.1023-1027, 1997

12. U. Pal, B. B. Chaudhuri, Script Line Separation from Indian Multi-Script Documents, Proceedings of fifth Intl. Conf. Document Analysis and Recognition, pp.406-409, 1999

13. U. Pal, B. B. Chaudhuri, Automatic Identification of English, Chinese, Arabic, Devnagari and Bangla Script Line, Intl. Conf. Document Analysis and Recognition, pp.0790-0794, 2001

14. U. Pal, S. Sinha, B. B. Chaudhuri, Multi-Script Line identification from Indian Documents, Proceedings of the Seventh International Conference on Document Analysis and Recognition, vol.2, pp.880-884, 2003

15. S. Chaudhury, R. Sheth, Trainable Script Identification Strategies for Indian Languages, Proceedings of 5th International Conference on Document Analysis and Recognation, pp.657-660, 1999

16. S. Kanoun, A. Ennaji, Y. LeCourtier, A. M. Alimi, Script and Nature Differentiation for Arabic and Latin Text Images, Proceedings of the 8th International Workshop on Frontiers in Handwriting Recognition, pp. 309-313, 2002 